TRANSACTIONS OF THE

AMERICAN MATHEMATICAL SOCIETY

Volume 355 , Number 4, Pages 1713-1728

S 0002-9947(02)03216-6

Article electronically published on December 2, 2002

\title{
PROJECTIVELY FLAT FINSLER METRICS OF CONSTANT FLAG CURVATURE
}

\author{
ZHONGMIN SHEN
}

\begin{abstract}
Finsler metrics on an open subset in $\mathrm{R}^{n}$ with straight geodesics are said to be projective. It is known that the flag curvature of any projective Finsler metric is a scalar function of tangent vectors (the flag curvature must be a constant if it is Riemannian). In this paper, we discuss the classification problem on projective Finsler metrics of constant flag curvature. We express them by a Taylor expansion or an algebraic formula. Many examples constructed in this paper can be used as models in Finsler geometry.
\end{abstract}

\section{INTRODUCTION}

Hilbert's Fourth Problem is to characterize the (not necessarily reversible) distance functions on an open subset in $\mathrm{R}^{n}$ such that straight lines are shortest paths Hi. It turns out that there are lots of solutions to the problem [Ha, [Bl, $\mathrm{Am}$, $[\mathrm{Al}], \mathrm{Bu}, \mathrm{Po}, \mathrm{Sz}$. The main method to characterize the solutions is the integral geometric construction using quasi-positive measures on the set of all affine hyperplanes in $\mathrm{R}^{n}$. The integral construction using symplectic forms gives another description on certain smooth solutions to the problem Alv1, Alv2, AlFe, AlGeSm.

Distance functions induced by Finsler metrics are regarded as smooth ones. Thus Hilbert's Fourth Problem in the smooth case is to characterize Finsler metrics on an open subset in $\mathrm{R}^{n}$ whose geodesics are straight lines. Such Finsler metrics are called projective Finsler metrics. In Finsler geometry, the flag curvature $\mathbf{K}(P, \mathbf{y})$ is an analogue of the sectional curvature in Riemannian geometry. It is known that every projective Finsler metric is of scalar curvature, namely, the flag curvature $\mathbf{K}(P, \mathbf{y})=\mathbf{K}(\mathbf{y})$ is a scalar function of tangent vectors $\mathbf{y}$. In the early 20 th century, L. Berwald studied projective Finsler metrics of constant flag curvature, particularly in the case of zero flag curvature [Be1], [Be2]. Berwald showed that the (projective) Hilbert metric on a strongly convex domain in $\mathrm{R}^{n}$ has constant flag curvature $\mathbf{K}=-1$. Meanwhile P. Funk classified all projective Finsler metrics with constant flag curvature on convex domains in $\mathrm{R}^{2}$ [Fk1], [Fk2]. Later on, Funk tried to show the uniqueness of projectively flat Finsler metrics with $\mathbf{K}=1$ on $\mathrm{S}^{2}$. With additional conditions, he showed that the standard Riemannian metric is the only such metric [Fk3]. In 1995-96, R. Bryant showed that there is exactly a 2-parameter family of projectively flat Finsler metrics on $\mathrm{S}^{2}$ with $\mathbf{K}=1[\mathrm{Br} 1]$, Br2].

Received by the editors July 1, 2002.

2000 Mathematics Subject Classification. Primary 53C60, 53A20.

(C)2002 American Mathematical Society 
Given a projective Finsler metric $F(x, y)$ on an open subset $\mathcal{U} \subset \mathrm{R}^{n}$, let

$$
P(x, y):=\frac{F_{x^{k}}(x, y) y^{k}}{2 F(x, y)} .
$$

It is known that the geodesics $c(t)=\left(x^{i}(t)\right)$ of $F$ are characterized by

$$
\frac{d^{2} x^{i}}{d t^{2}}+2 P\left(x, \frac{d x}{d t}\right) \frac{d x^{i}}{d t}=0 .
$$

See Lemma 3.1 below. We call $P(x, y)$ the projective factor of $F(x, y)$.

In this paper, we first give a general formula for $x$-analytic projective Finsler metrics $F(x, y)$ of constant flag curvature. Here $F(x, y)$ is said to be $x$-analytic at $x=0$ if there is a small number $\epsilon>0$ such that for any $y \neq 0, F(x, y)$ can be expressed as a power series $\sum a_{i_{1} \cdots i_{k}}(y) x^{i_{1}} \cdots x^{i_{k}}$ in $x$. For an $x$-analytic projective Finsler metric $F(x, y)$ at $x=0$, its projective factor $P(x, y)$ is $x$-analytic at $x=0$ too. We are going to show that both $F(x, y)$ and $P(x, y)$ are completely determined by $F(0, y)=\psi(y)$ and $P(0, y)=\varphi(y)$.

Theorem 1.1. Let $F(x, y)$ be a projective Finsler metric of constant flag curvature $\mathbf{K}=\lambda$ on an open neighborhood $\mathcal{U}$ of the origin in $\mathrm{R}^{n}$. Suppose that $F(x, y)$ is $x$ analytic at $x=0$. Then $F(x, y)$ and its projective factor $P(x, y)$ are given by

$$
\begin{aligned}
& F(x, y)=\left.\sum_{m=0}^{\infty} \frac{1}{(m+1) !} \frac{d^{m}}{d t^{m}}\left[\Psi_{m}(y+t x)\right]\right|_{t=0}, \\
& P(x, y)=\left.\sum_{m=0}^{\infty} \frac{1}{(m+1) !} \frac{d^{m}}{d t^{m}}\left[\Phi_{m}(y+t x)\right]\right|_{t=0}
\end{aligned}
$$

where

$$
\begin{gathered}
\Psi_{m}:= \begin{cases}\frac{1}{2 \sqrt{-\lambda}}\left\{(\varphi+\sqrt{-\lambda} \psi)^{m+1}-(\varphi-\sqrt{-\lambda} \psi)^{m+1}\right\} & \text { if } \lambda \neq 0, \\
(m+1) \psi \varphi^{m} & \text { if } \lambda=0,\end{cases} \\
\Phi_{m}:= \begin{cases}\frac{1}{2}\left\{(\varphi+\sqrt{-\lambda} \psi)^{m+1}+(\varphi-\sqrt{-\lambda} \psi)^{m+1}\right\} & \text { if } \lambda \neq 0, \\
\varphi^{m+1} & \text { if } \lambda=0,\end{cases}
\end{gathered}
$$

where $\psi(y):=F(0, y)$ and $\varphi(y):=P(0, y)$. Conversely, for any Minkowski norm $\psi(y)$ on $\mathrm{R}^{n}$ and any positively homogeneous function $\varphi(y)$ of degree one on $\mathrm{R}^{n}$, the function $F(x, y)$ defined in (1) is a projective Finsler metric with constant flag curvature $\mathbf{K}=\lambda$, and its projective factor $P(x, y)$ is given by (2) provided that they are convergent.

In [Be2], L. Berwald proved that for any projective Finsler metric $F(x, y)$ of flag curvature $\mathbf{K}=\lambda$ and its projective factor $P(x, y), F_{x^{k}}=(P F)_{y^{k}}$, and the function $f(x, y):=P(x, y) \pm \sqrt{-\lambda} F(x, y)$ satisfies

$$
f_{x^{k}}(x, y)=f(x, y) f_{y^{k}}(x, y) .
$$

By these equations, we prove Theorem 1.1 in Section 4 . Theorem [1.1 shows that $x$-analytic projective Finsler metrics of constant flag curvature $\mathbf{K}=\lambda$ are uniquely determined by $\psi(y)=F(0, y)$ and $\varphi(y)=P(0, y)$. Note that a Finsler metric $F(x, y)$ expressed in (11) is reversible if and only if $\psi(y)$ is reversible $(\psi(-y)=\psi(y))$ and $\varphi(y)$ is anti-reversible $(\varphi(-y)=-\varphi(y))$. Thus there are lots of reversible projective non-Riemannian Finsler metrics of constant flag curvature. 
We can construct smooth projective Finsler metrics of constant flag curvature using algebraic equations.

Theorem 1.2. Let $\psi(y)$ be an arbitrary Minkowski norm on $\mathrm{R}^{n}$ and $\varphi(y)$ be an arbitrary positively homogeneous function of degree one on $\mathrm{R}^{n}$. Define $\Psi_{ \pm}(x, y)$ by

$$
\Psi_{ \pm}(x, y)=\phi_{ \pm}\left(y+\Psi_{ \pm}(x, y) x\right)
$$

where $\phi_{ \pm}(y):=\varphi(y) \pm \psi(y)$. Then

$$
F(x, y):=\frac{1}{2}\left\{\Psi_{+}(x, y)-\Psi_{-}(x, y)\right\}
$$

is a projective Finsler metric with constant flag curvature $\mathbf{K}=-1$ and $F(0, y)=$ $\psi(y)$. Its projective factor $P(x, y)$ is given by

$$
P(x, y)=\frac{1}{2}\left\{\Psi_{+}(x, y)+\Psi_{-}(x, y)\right\}
$$

with $P(0, y)=\varphi(y)$.

The proof of Theorem 1.2 is given in Section [5 We first prove that the functions $\Psi_{ \pm}(x, y)$ defined in (4) satisfy (3), i.e., $\left(\Psi_{ \pm}\right)_{x^{k}}=\Psi_{ \pm}\left(\Psi_{ \pm}\right)_{y^{k}}$; then, using this equation, we show that the functions $F(x, y)$ and $P(x, y)$ defined in (5) and (6) have the desired properties. That is, we construct a projective Finsler metric of constant flag curvature $\mathbf{K}=-1$ using an algebraic equation (4) for any given data $\{\varphi(y), \psi(y)\}$. By the formulas in Theorem 1.2, we obtain many interesting examples.

Theorem 1.3. Let $\psi(y)$ be an arbitrary Minkowski norm on $\mathrm{R}^{n}$ and $\varphi(y)$ be an arbitrary positively homogeneous function of degree one on $\mathrm{R}^{n}$. Define $P(x, y)$ by

$$
P(x, y)=\varphi(y+P(x, y) x) .
$$

Let

$$
F(x, y):=\psi(y+P(x, y) x)\left\{1+P_{y^{m}}(x, y) x^{m}\right\} .
$$

Then $F(x, y)$ is a projective Finsler metric with zero flag curvature and $F(0, y)=$ $\psi(y)$, and its projective factor is $P(x, y)$ with $P(0, y)=\varphi(y)$.

The proof of Theorem 1.3 is given in Section 6. In [Be2], Berwald showed that any projective Finsler metric $F(x, y)$ of zero flag curvature and its projective factor, $P(x, y)$, satisfy $F_{x^{k}}=(P F)_{y^{k}}$ and $P_{x^{k}}=P P_{y^{k}}$. Then Berwald gave a formula in certain special cases. In particular, he constructed a nontrivial projective Finsler metric of zero flag curvature. See (49) below. In Theorem 1.3, we construct a projective Finsler metric of zero flag curvature for any given data $\{\varphi(y), \psi(y)\}$. By the formulas in Theorem 1.3, we obtain many interesting examples.

The construction of projective Finsler metrics of positive constant curvature is much more complicated. We will discuss this issue in Section 7

The above theorems only characterize the local metric structure of projective Finsler metrics of constant flag curvature. When we impose some extra global conditions, the projective Finsler metrics of constant curvature are much more special. For example, H. Akbar-Zadeh proves that any Finsler metric of negative constant flag curvature on a compact manifold must be Riemannian and any Finsler metric of zero curvature on a compact manifold is locally Minkowskian [AZ]. 
The Beltrami theorem says that a Riemannian metric is locally projectively flat if and only if it is of constant flag curvature. For Finsler metrics, this is no longer true. The first family of non-projectively-flat Finsler metrics of constant flag curvature was constructed in [BaSh]. Later on, the author constructed infinitely many non-projectively-flat Finsler metrics with constant flag curvature [Sh2], Sh3]. So far, many known non-projectively-flat Finsler metrics of constant flag curvature are in the form $F=\alpha+\beta$, where $\alpha$ is a Riemannian metric and $\beta$ is a 1 -form. Such metrics are called Randers metrics Ran. Recently, D. Bao and C. Robles BaRo have found an equivalent condition for Randers metrics to be of constant flag curvature. See also [MaSh. However, the classification problem for Randers metrics of constant flag curvature has not yet been completely solved.

After the preliminary version of this paper was sent out in the summer of 2001, the author received an interesting paper from R. Bryant [Br3], in which Bryant characterizes the (generalized) Finsler metrics on $\mathrm{S}^{n}$ with $\mathbf{K}=1$ and great circles as geodesics (see Theorem 2 in [Br3]). As argued by Bryant, the idea of Theorem 2 in [Br3] can be used to construct projective Finsler metrics with constant flag curvature $\mathbf{K}=1$ and a prescribed tangent indicatrix at one point (see Proposition 4 in $[\mathrm{Br} 3)$. He also shows how to construct all possible local projective Finsler metrics with $\mathbf{K}=1$. In a private communication, Bryant informed the author that his idea can also be used to characterize (local) projective Finsler metrics of constant flag curvature $\mathbf{K}=0$ or -1 , although he did not give any detailed discussion on this issue in [Br3]. Nevertheless, our results overlap Bryant's results in the positive flag curvature case. Here we only discuss the local construction, while Bryant in [Br3] discusses both the local and global issues. We should point out that our approach is different from Bryant's; hence the expressions of our examples are also different from his.

Many Finsler metrics with special curvature properties exist only locally. For example, there are lots of reversible projective Finsler metrics with $\mathbf{K}=1$ on an open subset of $\mathrm{S}^{n}$, which cannot be extended to the whole $\mathrm{S}^{n}$. R. Bryant shows that the only reversible projective Finsler metric with $\mathbf{K}=1$ on $\mathrm{S}^{2}$ is the standard Riemannian metric [Br1], [Br2]. I thank the referee for pointing out that the above problem in higher dimensions can be resolved by the discussion around Bryant's Example 2 in [Br3]. Namely, the only reversible projective Finsler metric with $\mathbf{K}=1$ on $\mathrm{S}^{n}$ is the standard Riemannian metric.

Acknowledgments. This work was done during the author's visit to Prof. S. S. Chern. It was presented at the 2nd National Conference on Finsler Geometry at the Nankai Institute of Mathematics on August 1, 2001. The author would like to thank S. S. Chern for his warm hospitality and valuable discussions, and also R. Bryant and Z. I. Szabó for explaining their work to the author.

\section{Preliminaries}

A Minkowski norm $\psi(y)$ on a vector space $\mathrm{V}$ is a $C^{\infty}$ function on $\mathrm{V} \backslash\{0\}$ with the following properties: (a) $\psi(y) \geq 0$, and $\psi(y)=0$ if and only if $y=0$; (b) $\psi(y)$ is a positively homogeneous function of degree one, i.e., $\psi(t y)=t \psi(y), t \geq 0 ;(\mathrm{c}) \psi(y)$ is strongly convex, i.e., for any $y \neq 0$, the matrix $g_{i j}(y):=\frac{1}{2}\left[F^{2}\right]_{y^{i} y^{j}}(y)$ is positive definite. A Finsler metric $F$ on a manifold $M$ is a $C^{\infty}$ function on $T M \backslash\{0\}$ such that $F_{x}:=\left.F\right|_{T_{x} M}$ is a Minkowski norm on $T_{x} M$ for any $x \in M$. 
Let $F$ be a Finsler metric on an $n$-dimensional manifold $M$. For a non-zero vector $\mathbf{y} \in T_{p} M, F$ induces an inner product $g_{\mathbf{y}}$ on $T_{p} M$ by

$$
g_{\mathbf{y}}(\mathbf{u}, \mathbf{v}):=g_{i j}(x, y) u^{i} v^{j}=\frac{1}{2}\left[F^{2}\right]_{y^{i} y^{j}}(x, y) u^{i} v^{j} .
$$

Here $x=\left(x^{i}\right)$ denotes the coordinates of $p \in M$ and $(x, y)=\left(x^{i}, y^{i}\right)$ denotes the local coordinates of $\mathbf{y} \in T_{p} M$. The geodesics are characterized by

$$
\frac{d^{2} x^{i}}{d t^{2}}+2 G^{i}\left(x(t), \frac{d x}{d t}(t)\right)=0,
$$

where $G^{i}:=\frac{1}{2} g^{i l}\left\{\left[F^{2}\right]_{x^{k}} y^{l} y^{k}-\left[F^{2}\right]_{x^{l}}\right\}$ are called the geodesic coefficients of $F$. $F$ is said to be positively complete (resp. complete) if every geodesic defined on $(a, b)$ can be extended to a geodesic defined on $(a, \infty)$ (resp. $(-\infty, \infty))$. Many examples found in this paper are positively complete, but not complete.

The Riemann curvature $\mathbf{R}_{\mathbf{y}}=\left.R_{k}^{i} d x^{k} \otimes \frac{\partial}{\partial x^{i}}\right|_{p}: T_{p} M \rightarrow T_{p} M$ is a family of linear maps on tangent spaces, defined by

$$
R_{k}^{i}=2 \frac{\partial G^{i}}{\partial x^{k}}-y^{j} \frac{\partial^{2} G^{i}}{\partial x^{j} \partial y^{k}}+2 G^{j} \frac{\partial^{2} G^{i}}{\partial y^{j} \partial y^{k}}-\frac{\partial G^{i}}{\partial y^{j}} \frac{\partial G^{j}}{\partial y^{k}} .
$$

For a tangent plane $P \subset T_{p} M$ and a non-zero vector $\mathbf{y} \in T_{p} M$, the flag curvature $\mathbf{K}(P, y)$ is defined by

$$
\mathbf{K}(P, \mathbf{y}):=\frac{g_{\mathbf{y}}\left(\mathbf{u}, \mathbf{R}_{\mathbf{y}}(\mathbf{u})\right)}{g_{\mathbf{y}}(\mathbf{y}, \mathbf{y}) g_{\mathbf{y}}(\mathbf{u}, \mathbf{u})-g_{\mathbf{y}}(\mathbf{y}, \mathbf{u})^{2}},
$$

where $P=\operatorname{span}\{\mathbf{y}, \mathbf{u}\}$. When $F$ is Riemannian, $\mathbf{K}(P, \mathbf{y})=\mathbf{K}(P)$ is independent of $\mathbf{y} \in P$. It is just the sectional curvature of $P$ in Riemannian geometry. We say that $F$ is of scalar curvature if for any $\mathbf{y} \in T_{p} M$, the flag curvature $\mathbf{K}(P, \mathbf{y})=\mathbf{K}(\mathbf{y})$ is independent of $P$ containing $\mathbf{y} \in T_{p} M$, which is equivalent to the following system of equations in a local coordinate system $\left(x^{i}, y^{i}\right)$ in $T M$ :

$$
R_{k}^{i}=\mathbf{K} F^{2}\left\{\delta_{k}^{i}-F^{-1} F_{y^{k}} y^{i}\right\} .
$$

If $\mathbf{K}$ is a constant, then $F$ is said to be of constant flag curvature.

There are several non-Riemannian quantities in Finsler geometry. One of the important non-Riemannian quantities is the E-curvature $\mathbf{E}_{\mathbf{y}}=\left.E_{i j} d x^{i} \otimes d x^{j}\right|_{p}$ : $T_{p} M \otimes T_{p} M \rightarrow \mathrm{R}$, defined by

$$
E_{i j}:=\frac{1}{2} \frac{\partial^{2}}{\partial y^{i} \partial y^{j}}\left[\frac{\partial G^{m}}{\partial y^{m}}\right]
$$

The E-curvature is closely related to the flag curvature. For a two-dimensional plane $P \subset T_{p} M$ and a non-zero vector $\mathbf{y} \in T_{p} M$, the flag E-curvature $\mathbf{E}(P, y)$ is defined by

$$
\mathbf{E}(P, \mathbf{y}):=\frac{F^{3}(\mathbf{y}) \mathbf{E}_{\mathbf{y}}(\mathbf{u}, \mathbf{u})}{g_{\mathbf{y}}(\mathbf{y}, \mathbf{y}) g_{\mathbf{y}}(\mathbf{u}, \mathbf{u})-g_{\mathbf{y}}(\mathbf{y}, \mathbf{u})^{2}},
$$

where $P=\operatorname{span}\{\mathbf{y}, \mathbf{u}\}$. We say that $F$ has constant flag E-curvature if for any flag $(P, \mathbf{y}), \mathbf{E}(P, \mathbf{y})=(n+1) c$, which is equivalent to the following system of equations,

$$
E_{i j}=(n+1) c F_{y^{i} y^{j}} .
$$

We know that the Funk metric on a strongly convex domain satisfies $\mathbf{K}=-1 / 4$ and $\mathbf{E}=(n+1) / 4$ Sh1] (see Example 5.3 below). There are many Finsler manifolds of constant flag curvature and constant flag E-curvature [Sh2], Sh3]. 


\section{Projective metrics}

A distance function $d$ on a manifold $M$ is said to be smooth if it is induced by a Finsler metric $F$ on $M$,

$$
d(p, q):=\inf _{c} \int_{0}^{1} F(\dot{c}(t)) d t
$$

where the infimum is taken over all curves $c(t), 0 \leq t \leq 1$, joining $p=c(0)$ to $q=c(1)$. Thus smooth distance functions can be studied using calculus (see [AIM], [BCS]). Hilbert's Fourth Problem in the smooth case is to characterize Finsler metrics on an open subset in $\mathrm{R}^{n}$ whose geodesics are straight lines.

Let $\mathcal{U} \subset \mathrm{R}^{n}$ be an open subset. A Finsler metric on $\mathcal{U}$ is a function on $T \mathcal{U} \cong$ $\mathcal{U} \times \mathrm{R}^{n}$. It is known that a Finsler metric $F(x, y)$ on $\mathcal{U}$ is projective if and only if its geodesic coefficients $G^{i}$ are in the form

$$
G^{i}(x, y)=P(x, y) y^{i},
$$

where $P(x, y)$ is positively $y$-homogeneous with degree one, $P(x, k y)=k P(x, y)$, $k>0$. We call $P(x, y)$ the projective factor of $F(x, y)$. The following lemma plays an important role.

Lemma 3.1 ( $\mathrm{Ha}$, Rap $)$. Let $F(x, y)$ be a Finsler metric on an open subset $\mathcal{U} \subset \mathrm{R}^{n} . F(x, y)$ is projective on $\mathcal{U}$ if and only if it satisfies

$$
F_{x^{k} y^{l}}(x, y) y^{k}=F_{x^{l}}(x, y) .
$$

In this case, the projective factor $P(x, y)$ is given by

$$
P(x, y)=\frac{F_{x^{k}}(x, y) y^{k}}{2 F(x, y)} .
$$

Let $F(x, y)$ be a projective Finsler metric on an open subset $\mathcal{U} \subset \mathrm{R}^{n}$, and let $P(x, y)$ denote its projective factor. Plugging $G^{i}=P y^{i}$ into (9) yields

$$
R_{k}^{i}=\Xi \delta_{k}^{i}+\tau_{k} y^{i}
$$

where

$$
\Xi:=P^{2}-P_{x^{k}} y^{k}, \quad \tau_{k}:=3\left(P_{x^{k}}-P P_{y^{k}}\right)+\Xi_{y^{k}} .
$$

By the symmetry property that $g_{j i} R_{k}^{i}=g_{k i} R_{j}^{i}$, we obtain

$$
R_{k}^{i}=\Xi\left\{\delta_{k}^{i}-F^{-1} F_{y^{k}} y^{i}\right\} \text {. }
$$

Thus

$$
P_{x^{k}}-P P_{y^{k}}=-\frac{(\Xi F)_{y^{k}}}{3 F} .
$$

From (17), we see that $F$ is of scalar curvature with flag curvature

$$
\mathbf{K}=\Xi / F^{2} \text {. }
$$

This observation was first made by L. Berwald $\mathrm{Be} 2$. The following lemma follows from his arguments. 
Lemma 3.2. Let $F(x, y)$ be a Finsler metric on an open subset $\mathcal{U} \subset \mathrm{R}^{n}$. Then $F=F(x, y)$ is projective with flag curvature $\mathbf{K}=\mathbf{K}(x, y)$ if and only if there are a positively y-homogeneous function $P=P(x, y)$ of degree one and a positively homogeneous function $\mathbf{K}=\mathbf{K}(x, y)$ of degree zero on $T \mathcal{U} \cong \mathcal{U} \times \mathrm{R}^{n}$ such that

$$
F_{x^{k}}=(P F)_{y^{k}}
$$

and

$$
P_{x^{k}}=P P_{y^{k}}-\frac{1}{3 F}\left(\mathbf{K} F^{3}\right)_{y^{k}} .
$$

In this case, $P$ is the projective factor of $F$.

\section{Analytic solutions}

In this section, we are going to determine the local structures of $x$-analytic projective Finsler metrics $F(x, y)$ of constant flag curvature by solving the firstorder partial differential equations (201) and (21).

Let $F(x, y)$ be a projective Finsler metric of constant flag curvature $\mathbf{K}=\lambda$ on a neighborhood of the origin in $\mathrm{R}^{n}$, and $P(x, y)$ be the projector factor. It follows from Lemma 3.2 that

$$
F_{x^{k}}=(P F)_{y^{k}}, \quad P_{x^{k}}=P P_{y^{k}}-\lambda F F_{y^{k}} .
$$

Let $\Psi_{m}(y)$ and $\Phi_{m}(y)$ be defined as in Theorem 1.1.

Assume that $\lambda \neq 0$. Let $\Psi_{ \pm}(x, y):=P(x, y) \pm \sqrt{-\lambda} F(x, y)$. Then we can express $F(x, y)$ and $P(x, y)$ in terms of $\Psi_{ \pm}(x, y)$. It follows from (22) that

$$
\left(\Psi_{ \pm}\right)_{x^{k}}=\Psi_{ \pm}\left(\Psi_{ \pm}\right)_{y^{k}}
$$

This observation is due to Berwald $\mathrm{Be} 2$.

Now we are going to use (22) and (23) to find the Taylor expansion for $x$-analytic projective Finsler metrics of constant flag curvature $\mathbf{K}=\lambda \neq 0$.

Differentiating $\Psi_{ \pm}(x, y)$ and using (23), we obtain

$$
\left(\Psi_{ \pm}\right)_{x^{i_{1} \ldots x^{i} m}}(0, y)=\frac{1}{m+1}\left[(\varphi \pm \sqrt{-\lambda} \psi)^{m+1}\right]_{y^{i_{1} \ldots y^{i_{m}}}}(y) .
$$

By (24) we obtain

$$
\begin{aligned}
& F_{x^{i_{1} \ldots x^{i_{m}}}}(0, y)=\frac{1}{(m+1)}\left[\Psi_{m}\right]_{y^{i_{1} \ldots y^{i_{m}}}}(y), \\
& P_{x^{i_{1} \ldots x^{i_{m}}}}(0, y)=\frac{1}{(m+1)}\left[\Phi_{m}\right]_{y^{i_{1} \ldots y^{i_{m}}}}(y) .
\end{aligned}
$$

By (25) and (26), we immediately obtain (12) and (2).

Now assume that $\lambda=0$. It follows from $(22)$ that

$$
F_{x^{k}}=(P F)_{y^{k}}, \quad P_{x^{k}}=P P_{y^{k}} .
$$

By the first equation of (27), we have

$$
F_{x^{i_{1} \ldots x^{i_{m}}}}(x, y)=\left(F P^{m}\right)_{y^{i_{1} \cdots y^{i_{m}}}}(x, y) .
$$

Setting $x=0$ in (28) yields

$$
F_{x^{i_{1} \ldots x^{i m}}}(0, y)=\frac{1}{(m+1)}\left[\Psi_{m}\right]_{y^{i_{1} \ldots y^{i_{m}}}}(y) .
$$


Since $P(x, y)$ satisfies the second equation of (27) with $P(0, y)=\varphi(y)$, we have

$$
P_{x^{i_{1} \ldots x^{i_{m}}}}(0, y)=\frac{1}{m+1}\left[\Phi_{m}\right]_{y^{i_{1} \ldots y^{i_{m}}}}(y) .
$$

By the above identities, we obtain (11) and (2) in the case when $\lambda=0$.

The second part of the theorem is left for the reader to verify.

$$
\text { 5. } \mathbf{K}=-1
$$

In this section, we are going to construct smooth solutions $F(x, y)$ of constant flag curvature $\mathbf{K}=-1$ with $F(0, y)=\psi(y)$ and $F_{x^{k}}(0, y) y^{k}=2 \psi(y) \varphi(y)$ for any given pair $\{\psi(y), \varphi(y)\}$. We need the following lemma.

Lemma 5.1. Let $\phi(y)$ be an arbitrary positively homogeneous function of degree one on $\mathrm{R}^{n}$. Suppose that $\phi$ is $C^{\infty}$ on $\mathrm{R}^{n} \backslash\{0\}$. There is a unique real-valued function $f:=f(x, y)$ satisfying the following:

$$
f(x, y)=\phi(y+f(x, y) x) .
$$

Moreover, $f$ satisfies

$$
f_{x^{k}}=f f_{y^{k}} .
$$

Proof. Let $h(t):=t-\phi(y+t x)$. Observe that there is a small $\delta>0$ such that for any $x \in \mathrm{R}^{n}$ with $|x|<\delta$, at any $t$ where $y+t x \neq 0$,

$$
h^{\prime}(t)=1-\phi_{y^{i}}(y+t x) x^{k} \geq \frac{1}{2} .
$$

Thus there is a unique $t_{o}$ such that $h\left(t_{o}\right)=0$. Setting $f(x, y):=t_{o}$, we obtain the unique solution.

Differentiating (29) with respect to $x^{k}$ and $y^{k}$, respectively, we obtain

$$
\begin{aligned}
& \left(1-\phi_{y^{m}} x^{m}\right) f_{x^{k}}=\phi_{y^{k}} f, \\
& \left(1-\phi_{y^{m}} x^{m}\right) f_{y^{k}}=\phi_{y^{k}} .
\end{aligned}
$$

For $x$ close to $0, \phi_{y^{m}} x^{m}<1$. Thus (30) holds.

Proof of Theorem 1.2. Let $\Psi_{ \pm}(x, y)$ be the functions defined in (4). By Lemma 5.1, $\Psi_{ \pm}=\Psi_{ \pm}(x, y)$ satisfy (30), i.e.,

$$
\left(\Psi_{ \pm}\right)_{x^{k}}=\Psi_{ \pm}\left(\Psi_{ \pm}\right)_{y^{k}}
$$

with $\Psi_{ \pm}(0, y)=\phi_{ \pm}(y)=\varphi(y) \pm \psi(y)$. Let $F:=\frac{1}{2}\left\{\Psi_{+}-\Psi_{-}\right\}$be defined in (5). It follows from (31) that

$$
\left(\Psi_{ \pm}\right)_{x^{k} y^{l}} y^{k}=\left(\Psi_{ \pm}\right)_{x^{l}}
$$

Thus $F(x, y)$ satisfies (13), and it is projective by Lemma 3.1. Observe that

$$
F_{x^{k}} y^{k}=\frac{1}{2}\left\{\Psi_{+}\left(\Psi_{+}\right)_{y^{k}}-\Psi_{-}\left(\Psi_{-}\right)_{y^{k}}\right\} y^{k}=\frac{1}{2}\left\{\Psi_{+}^{2}-\Psi_{-}^{2}\right\} .
$$

Thus the projective factor $P=\frac{1}{2} F^{-1} F_{x^{k}} y^{k}$ is given by

$$
P:=\frac{F_{x^{k}} y^{k}}{2 F}=\frac{1}{2} \frac{\Psi_{+}^{2}-\Psi_{-}^{2}}{\Psi_{+}-\Psi_{-}}=\frac{1}{2}\left\{\Psi_{+}+\Psi_{-}\right\}
$$


By a similar argument, we obtain

$$
\Xi=P^{2}-P_{x^{k}} y^{k}=-\left(\frac{\Psi_{+}-\Psi_{-}}{2}\right)^{2}=-F^{2} .
$$

Thus the flag curvature $\mathbf{K}=-1$ by (19).

Example 5.1. Let $\phi(y)$ be a Minkowski norm on $\mathrm{R}^{n}$ and $\mathcal{U}:=\left\{y \in \mathrm{R}^{n} \mid \phi(y)<1\right\}$. Define $\Theta(x, y)$ by

$$
\Theta(x, y)=\phi(y+\Theta(x, y) x), \quad y \in T_{x} \mathcal{U}
$$

$\Theta(x, y)$ is a Finsler metric on $\mathcal{U}$ satisfying the following system of equations $\mathrm{Ok}$ :

$$
\Theta_{x^{k}}=\Theta \Theta_{y^{k}} .
$$

By Lemma 3.2 $F(x, y):=\Theta(x, y)$ is a projective Finsler metric on $\mathcal{U}$ with constant flag curvature $\mathbf{K}=-1 / 4$, and its projective factor is given by $P(x, y):=\frac{1}{2} \Theta(x, y)$.

Let $\phi_{+}=\phi$, and let $\phi_{-}$be an arbitrary positively $y$-homogeneous function of degree one on $\mathrm{R}^{n}$. Let

$$
\varphi(y):=\frac{1}{2}\left\{\phi_{+}(y)+\phi_{-}(y)\right\}, \quad \psi(y):=\frac{1}{2}\left\{\phi_{+}(y)-\phi_{-}(y)\right\} .
$$

Let $\Psi_{ \pm}(x, y)$ be the solutions of (4). Let $F(x, y)$ and $P(x, y)$ be the functions defined in (5) and (6) using $\Psi_{ \pm}(x, y)$. By Theorem 1.2, $F(x, y)$ is projective with $\mathbf{K}=-1$, and its projective factor is $P(x, y)$.

Note that $\Psi_{+}(x, y)=\Theta(x, y)$. If $\phi_{-}(y):=-\phi(-y)$, then $\Psi_{-}(x, y)=-\Theta(x,-y)$. In this case,

$$
\begin{aligned}
& F(x, y)=\frac{1}{2}\{\Theta(x, y)+\Theta(x,-y)\}, \\
& P(x, y)=\frac{1}{2}\{\Theta(x, y)-\Theta(x,-y)\} .
\end{aligned}
$$

We call $F$ the Hilbert metric on $\mathcal{U}$.

Example 5.2. Let $\phi(y)$ be an arbitrary Minkowski norm on $\mathrm{R}^{n}$, and let $\Theta(x, y)$ denote the Funk metric of $\phi$ defined by (32). Let $\delta \neq 0$ and $\lambda$ be constants, and

$$
\psi(y):=\frac{1}{2}\{\phi(y)-\delta \phi(\lambda y)\}, \quad \varphi(y):=\frac{1}{2}\{\phi(y)+\delta \phi(\lambda y)\} .
$$

We assume that $\delta$ and $\lambda$ are such that $\psi(y)$ is a Minkowski norm on $\mathrm{R}^{n}$. We have

$$
(\varphi+\psi)(y)=\phi(y), \quad(\varphi-\psi)(y)=\delta \phi(\lambda y) .
$$

Let $\Psi_{+}(x, y)$ and $\Psi_{-}(x, y)$ be defined in (4) with $\phi_{+}(y)=\phi(y)$ and $\phi_{-}(y):=$ $\delta \phi(\lambda y)$, respectively. Then

$$
\Psi_{+}(x, y)=\Theta(x, y), \quad \Psi_{-}(x, y)=\delta \Theta(\delta \lambda x, \lambda y) .
$$

By Theorem 1.2, we conclude that the function

$$
F(x, y):=\frac{1}{2}\{\Theta(x, y)-\delta \Theta(\delta \lambda x, \lambda y)\}
$$

is a projective Finsler metric on its domain with $\mathbf{K}=-1$, and its projective factor is given by

$$
P(x, y)=\frac{1}{2}\{\Theta(x, y)+\delta \Theta(\delta \lambda x, \lambda y)\} .
$$

By taking $\delta=-1$ and $\lambda=-1$ in (36), we obtain the Hilbert metric. 
Take a look at the special case when $\phi(y)=|y|$, the Funk metric on $\mathrm{B}^{n}$, is given by

$$
\Theta(x, y)=\frac{\sqrt{|y|^{2}-\left(|x|^{2}|y|^{2}-\langle x, y\rangle^{2}\right)}+\langle x, y\rangle}{1-|x|^{2}}, \quad y \in T_{x} \mathrm{~B}^{n} .
$$

Let $\epsilon:=\delta|\lambda|$. Then the Finsler metric defined in (36) is given by

$$
\begin{aligned}
F(x, y)= & \frac{1}{2}\left\{\frac{\sqrt{|y|^{2}-\left(|x|^{2}|y|^{2}-\langle x, y\rangle^{2}\right)}+\langle x, y\rangle}{1-|x|^{2}}\right. \\
& \left.-\frac{\epsilon \sqrt{|y|^{2}-\epsilon^{2}\left(|x|^{2}|y|^{2}-\langle x, y\rangle^{2}\right)}+\epsilon^{2}\langle x, y\rangle}{1-\epsilon^{2}|x|^{2}}\right\} .
\end{aligned}
$$

Clearly, $F$ is positively complete on $\mathrm{B}^{n}(1)$ if $|\epsilon|<1$, and $F$ is the Klein metric when $\epsilon=-1$. By the above argument, we know that $F$ is projective with constant flag curvature $\mathbf{K}=-1$.

Example 5.3. Let $\phi(y)$ be an arbitrary Minkowski norm on $\mathbf{R}^{n}$, and let $\Theta(x, y)$ denote the Funk metric of $\phi$. For a constant vector $a \in \mathrm{R}^{n}$, let

$$
\psi(y):=\frac{1}{2}(\phi(y)+\langle a, y\rangle), \quad \varphi(y):=\frac{1}{2}(\phi(y)-\langle a, y\rangle)
$$

be such that

$$
\varphi(y)+\psi(y)=\phi(y), \quad \varphi(y)-\psi(y)=-\langle a, y\rangle .
$$

Let $\Psi_{+}(x, y)$ and $\Psi_{-}(x, y)$ be the functions defined by (4) with $\phi_{+}(y)=\phi(y)$ and $\phi_{-}(y)=-\langle a, y\rangle$, respectively. We have

$$
\Psi_{+}(x, y)=\Theta(x, y), \quad \Psi_{-}(x, y)=-\frac{\langle a, y\rangle}{1+\langle a, x\rangle} .
$$

By Theorem 1.2, we know that the function

$$
F(x, y)=\frac{1}{2}\left\{\Theta(x, y)+\frac{\langle a, y\rangle}{1+\langle a, x\rangle}\right\}
$$

is a projective Finsler metric with $\mathbf{K}=-1$, and its projective factor is given by

$$
P(x, y)=\frac{1}{2}\left\{\Theta(x, y)-\frac{\langle a, y\rangle}{1+\langle a, x\rangle}\right\} .
$$

Since the geodesic coefficients are in the form $G^{i}=P y^{i}$, a direct computation gives

$$
\frac{\partial G^{m}}{\partial y^{m}}=(n+1) P \text {. }
$$

Thus

$$
E_{i j}=\frac{1}{2} \frac{\partial^{2}}{\partial y^{i} \partial y^{j}}\left[\frac{\partial G^{m}}{\partial y^{m}}\right]=\frac{n+1}{2} P_{y^{i} y^{j}} .
$$

By (33) again, one immediately obtains

$$
P(x, y)=\frac{1}{2}\left\{\Theta(x, y)-\frac{\langle a, y\rangle}{1+\langle a, x\rangle}\right\} .
$$


Observe that

$$
\begin{aligned}
E_{i j} & =\frac{n+1}{4}\left[\Theta-\frac{\langle a, y\rangle}{1+\langle a, x\rangle}\right]_{y^{i} y^{j}} \\
& =\frac{n+1}{4}\left[\Theta+\frac{\langle a, y\rangle}{1+\langle a, x\rangle}\right]_{y^{i} y^{j}} \\
& =\frac{n+1}{2} F_{y^{i} y^{j}} .
\end{aligned}
$$

Thus the Finsler metric $F$ in (40) also has constant flag E-curvature, $\mathbf{E}=\frac{1}{2}(n+1)$.

Take a look at the special case when $\phi(y)=|y|$. The Funk metric $\Theta(x, y)$ on the unit ball $\mathrm{B}^{n}(1)$ is given by (38). Thus for any constant vector $a \in \mathrm{R}^{n}$ with $|a|<1$, the function

$$
F(x, y)=\frac{1}{2}\left\{\frac{\sqrt{|y|^{2}-\left(|x|^{2}|y|^{2}-\langle x, y\rangle^{2}\right)}+\langle x, y\rangle}{1-|x|^{2}}+\frac{\langle a, y\rangle}{1+\langle a, x\rangle}\right\}
$$

is a projective Finsler metric on $\mathrm{B}^{n}(1)$ with $\mathbf{K}=-1$ and $\mathbf{E}=\frac{1}{2}(n+1)$. In [Sh4], we prove that a Randers metric is projective with constant flag curvature if and only if it is locally Minkowskian or, up to a scaling, isometric to the metric in (42).

$$
\text { 6. } \mathbf{K}=0
$$

In this section, we are going to prove Theorem 1.3, and then construct some interesting projective Finsler metrics $F(x, y)$ with zero flag curvature $\mathbf{K}=0$.

Proof of Theorem 1.3. Let $P(x, y)$ and $F(x, y)$ be defined in (7) and (8) respectively. By Lemma 5.1, $P(x, y)$ satisfies

$$
P_{x^{k}}=P P_{y^{k}}
$$

with $P(0, y)=\varphi(y)$. It follows from (43) that

$$
P_{x^{k} y^{m}}=\frac{1}{2}\left(P^{2}\right)_{y^{k} y^{m}}=P_{x^{m} y^{k}}
$$

Differentiating (8) with respect to $x^{k}$ and using (43), we obtain

$$
\begin{aligned}
F_{x^{k}}= & \psi_{y^{m}}(y+P x)\left\{P \delta_{k}^{m}+P_{x^{k}} x^{m}\right\}\left\{1+P_{y^{l}} x^{l}\right\} \\
& +\psi(y+P x)\left\{P_{y^{k}}+P_{y^{m} x^{k}} x^{m}\right\} .
\end{aligned}
$$

It follows from (43) and (8) that

$$
P F=\psi(y+P x)\left\{P+P_{x^{m}} x^{m}\right\} .
$$

Differentiating (45) with respect to $y^{k}$ yields

$$
\begin{aligned}
(P F)_{y^{k}}= & \psi_{y^{m}}(y+P x)\left\{\delta_{k}^{m}+P_{y^{k}} x^{m}\right\}\left\{P+P_{x^{l}} x^{l}\right\} \\
& +\psi(y+P x)\left\{P_{y^{k}}+P_{x^{m} y^{k}} x^{m}\right\} .
\end{aligned}
$$

By (43), we have

$$
\left\{P \delta_{k}^{m}+P_{x^{k}} x^{m}\right\}\left\{1+P_{y^{l}} x^{l}\right\}=\left\{\delta_{k}^{m}+P_{y^{k}} x^{m}\right\}\left\{P+P_{x^{l}} x^{l}\right\} .
$$

In virtue of (44), we conclude that $F$ satisfies

$$
F_{x^{k}}=(P F)_{y^{k}} .
$$


By Lemma 3.2, we conclude that the function $F(x, y)$ is a projective Finsler metric on its domain with $\mathbf{K}=0$, and its projective factor is $P(x, y)$.

Example 6.1. Let $\phi(y)$ be a Minkowski norm on $\mathrm{R}^{n}$, and let $\Theta(x, y)$ denote the Funk metric on $\mathcal{U}:=\left\{y \in \mathrm{R}^{n} \mid \phi(y)<1\right\}$, which is defined in (32). Let $\psi(y)=\phi(y)+\langle a, y\rangle$ and $\varphi(y)=\phi(y)$. Let $P(x, y)$ and $F(x, y)$ be defined in (7) and (8) respectively. We have

$$
P(x, y)=\Theta(x, y)
$$

Observe that

$$
\phi(y+P x)=\phi(y+\Theta x)=\Theta .
$$

Thus

$$
\psi(y+P x)=\Theta+\langle a, y\rangle+\langle a, x\rangle \Theta .
$$

This gives

$$
F(x, y)=\{(1+\langle a, x\rangle) \Theta(x, y)+\langle a, y\rangle\}\left\{1+\Theta_{y^{k}}(x, y) x^{k}\right\} .
$$

Take $\psi(y)=|y|+\langle a, y\rangle$ and $\varphi(y)=|y|$. We obtain

$$
\begin{aligned}
F(x, y)= & \left\{1+\langle a, x\rangle+\frac{\left(1-|x|^{2}\right)\langle a, y\rangle}{\sqrt{|y|^{2}-\left(|x|^{2}|y|^{2}-\langle x, y\rangle^{2}\right)}+\langle x, y\rangle}\right\} \\
& \times \frac{\left(\sqrt{|y|^{2}-\left(|x|^{2}|y|^{2}-\langle x, y\rangle^{2}\right.}+\langle x, y\rangle\right)^{2}}{\left(1-|x|^{2}\right)^{2} \sqrt{|y|^{2}-\left(|x|^{2}|y|^{2}-\langle x, y\rangle^{2}\right)}} .
\end{aligned}
$$

By Theorem 1.3. we know that $F(x, y)$ is a projective Finsler metric with zero flag curvature $\mathbf{K}=0$, and its projective factor is given by

$$
P(x, y)=\frac{\sqrt{|y|^{2}-\left(|x|^{2}|y|^{2}-\langle x, y\rangle^{2}\right)}+\langle x, y\rangle}{1-|x|^{2}} .
$$

Clearly, $F(x, y)$ is not locally Minkowskian.

Remark 6.1. Letting $a=0$ in (47), we obtain

$$
F(x, y)=\frac{\left(\sqrt{|y|^{2}-\left(|x|^{2}|y|^{2}-\langle x, y\rangle^{2}\right)}+\langle x, y\rangle\right)^{2}}{\left(1-|x|^{2}\right)^{2} \sqrt{|y|^{2}-\left(|x|^{2}|y|^{2}-\langle x, y\rangle^{2}\right)}} .
$$

This is just the projective Finsler metric constructed by L. Berwald [Be2]. The Finsler metric $F(x, y)$ in (49) is positively complete, i.e., every unit speed geodesic on $(\delta, \tau)$ can be extended to a geodesic on $(\delta, \infty)$. It can be easily shown that any complete projective Finsler metric with zero flag curvature $\mathbf{K}=0$ must be a Minkowski metric [Be1], Fk1].

Example 6.2. Let $\phi$ be an arbitrary Minkowski norm on $\mathrm{R}^{n}$. Take

$$
\psi(y):=\phi(y), \quad \varphi(y):=-\langle a, y\rangle .
$$

We obtain

$$
\begin{aligned}
P(x, y) & =-\frac{\langle a, y\rangle}{1+\langle a, x\rangle} \\
F(x, y) & =\frac{\phi((1+\langle a, x\rangle) y-\langle a, y\rangle x)}{(1+\langle a, x\rangle)^{2}} .
\end{aligned}
$$


By Theorem 1.3 we know that $F(x, y)$ is a projective Finsler metric on its domain with $\mathbf{K}=0$, and its projective factor is $P(x, y)$.

For the Finsler metric $F(x, y)$ in (51), the geodesic coefficients

$$
G^{i}(x, y)=P(x, y) y^{i}
$$

are quadratic in $y \in \mathrm{R}^{n}$. Thus $F$ is a Berwald metric. It is well known that any Berwald metric with $\mathbf{K}=0$ is locally Minkowskian. We conclude that the Finsler metric in (51) is locally Minkowskian.

Take a Randers norm, $\phi(y)=|y|+\langle b, y\rangle$, where $b \in \mathrm{R}^{n}$ is a vector with $|b|<1$. We obtain a projective Randers metric with $\mathbf{K}=0$,

$$
\begin{aligned}
F(x, y)= & \frac{\sqrt{(1+\langle a, x\rangle)^{2}|y|^{2}-2(1+\langle a, x\rangle)\langle a, y\rangle\langle x, y\rangle+\langle a, y\rangle^{2}|x|^{2}}}{(1+\langle a, x\rangle)^{2}} \\
& +\frac{(1+\langle a, x\rangle)\langle b, y\rangle-\langle a, y\rangle\langle b, x\rangle}{(1+\langle a, x\rangle)^{2}} .
\end{aligned}
$$

This metric must be locally Minkowskian. This fact also follows from the main theorem in Sh4.

$$
\text { 7. } \mathbf{K}=1
$$

In this section, we are going to construct projective Finsler metrics $F(x, y)$ with constant flag curvature $\mathbf{K}=1$. Let $\psi(y)$ be a Minkowski norm on $\mathrm{R}^{n}$ and $\varphi(y)$ a positively homogeneous function of degree one on $\mathrm{R}^{n}$. We would like to find a projective Finsler metric $F(x, y)$ of constant flag curvature $\mathbf{K}=1$ satisfying $F(0, y)=\psi(y)$ and with projective factor $P(0, y)=\varphi(y)$. Suppose that there is a complex-valued solution to the following system:

$$
H_{x^{k}}=H H_{y^{k}}
$$

with $H(0, y)=\varphi(y)+i \psi(y)$. We can express $H(x, y)=P(x, y)+i F(x, y)$. Plugging it into (52) yields

$$
P_{x^{k}}-P P_{y^{k}}+F F_{y^{k}}+i\left\{F_{x^{k}}-(P F)_{y^{k}}\right\}=0 .
$$

We obtain

$$
F_{x^{k}}=(P F)_{y^{k}}, \quad P_{x^{k}}=P P_{y^{k}}-F F_{y^{k}} .
$$

By Lemma 3.2, $F(x, y)$ is projective with constant flag curvature $\mathbf{K}=1$ and with $F(0, y)=\psi(y)$, and its projective factor is $P(x, y)$ with $P(0, y)=\varphi(y)$.

Let

$$
H(x, y):=\left.\sum_{m=0}^{\infty} \frac{1}{(m+1) !} \frac{d^{m}}{d t^{m}}\left[(\varphi(y+t x)+i \psi(y+t x))^{m+1}\right]\right|_{t=0} .
$$

We assume that the above power series is convergent for $x \in \mathrm{R}^{n}$ close to the origin and any $y \in \mathrm{R}^{n}$. By a direct argument, one can verify that the function $H=H(x, y)$ in (53) satisfies equation (52) with $H(0, y)=\varphi(y)+i \psi(y)$.

Assume that $\phi(y):=\varphi(y)+i \psi(y)$ is $y$-analytic on $\mathrm{R}^{n} \backslash\{0\}$, so that for any $y \in \mathrm{R}^{n} \backslash\{0\}$, it can be extended to a function $\phi(y+z x), z \in \mathbb{C}$ and $x \in \mathrm{R}^{n}$, such that there is a solution $H(x, y)$ to the following equation:

$$
H(x, y)=\phi(y+H(x, y) x) .
$$


Note that $H(0, y)=\phi(y)$. By the same argument as in Lemma 5.1 one can show that $H=H(x, y)$ satisfies equation (52) with $H(0, y)=\varphi(y)+i \psi(y)$.

Example 7.1. Let $\psi(y)=\cos (\alpha)|y|$ and $\varphi(y)=\sin (\alpha)|y|$, where $|\cdot|$ denotes the Euclidean norm on $\mathrm{R}^{n}$ and $\alpha$ is an angle with $|\alpha|<\pi / 2$. We have

$$
\varphi(y)+i \psi(y)=i e^{-i \alpha}|y| .
$$

Equation (54) becomes

$$
H=i e^{-i \alpha} \sqrt{|y|^{2}+2\langle x, y\rangle H+|x|^{2} H^{2}} .
$$

We obtain

$$
H=\frac{-\langle x, y\rangle+i \sqrt{\left.\left(e^{2 i \alpha}+|x|^{2}\right\rangle\right)|y|^{2}-\langle x, y\rangle^{2}}}{e^{2 i \alpha}+|x|^{2}} .
$$

Express

$$
H(x, y)=P(x, y)+i F(x, y) .
$$

Then $F(x, y)$ is a projective Finsler metric of constant flag curvature $\mathbf{K}=1$, and $P(x, y)$ is the projective factor of $F(x, y)$. Let

$$
\begin{aligned}
A: & =\left(\cos (2 \alpha)|y|^{2}+\left(|x|^{2}|y|^{2}-\langle x, y\rangle^{2}\right)\right)^{2}+\left(\sin (2 \alpha)|y|^{2}\right)^{2} \\
B: & =\cos (2 \alpha)|y|^{2}+\left(|x|^{2}|y|^{2}-\langle x, y\rangle^{2}\right) \\
C: & =\sin (2 \alpha)\langle x, y\rangle \\
C^{\prime}: & =\left(\cos (2 \alpha)+|x|^{2}\right)\langle x, y\rangle \\
D: & =|x|^{4}+2 \cos (2 \alpha)|x|^{2}+1
\end{aligned}
$$

For an angle $\alpha$ with $0 \leq \alpha<\pi / 2$,

$$
\sqrt{\left(e^{2 \alpha i}+|x|^{2}\right)|y|^{2}-\langle x, y\rangle^{2}}=\sqrt{\frac{\sqrt{A}+B}{2}}+i \sqrt{\frac{\sqrt{A}-B}{2}} .
$$

By an elementary argument we obtain

$$
\begin{aligned}
& F(x, y)=\sqrt{\frac{\sqrt{A}+B}{2 D}+\left(\frac{C}{D}\right)^{2}}+\frac{C}{D}, \\
& P(x, y)=-\sqrt{\frac{\sqrt{A}-B}{2 D}-\left(\frac{C}{D}\right)^{2}}-\frac{C^{\prime}}{D} .
\end{aligned}
$$

In dimension two, one can verify that the Finsler metric $F$ in (56) is the Bryant metric [Br1], [Br2].

We can generalize the above construction. Let $\phi(y)$ be an arbitrary Minkowski norm on $\mathrm{R}^{n}$, and let $\Theta(x, y)$ denote the Funk metric of $\phi$. Let

$$
\psi(y):=\cos (\alpha) \phi(y), \quad \varphi(y):=\sin (\alpha) \phi(y),
$$

where $\alpha$ is an angle with $|\alpha|<\pi / 2$. We have

$$
\varphi(y)+i \psi(y)=i e^{-i \alpha} \phi(y) .
$$

Equation (54) becomes

$$
H=i e^{-i \alpha} \phi(y+H x) .
$$


Assume that the Funk metric $\Theta(x, y)$ is $x$-analytic in $x \in \mathrm{R}^{n}$ at $x=0$, so that it can be extended to be a function $\Theta(z x, y)$, where $z \in \mathbb{C}$ with $|z|=1$, and $y \in \mathrm{R}^{n}$ and $x \in \mathrm{R}^{n}$ are close to the origin. Then

$$
H(x, y):=i e^{-i \alpha} \Theta\left(i e^{-i \alpha} x, y\right)
$$

is a solution to (58) with $H(0, y)=i e^{-i \alpha} \phi(y)=\varphi(y)+i \psi(y)$. By the above argument, we know that the function

$$
F(x, y):=\frac{1}{2 i}\left\{i e^{-i \alpha} \Theta\left(i e^{-i \alpha} x, y\right)-\overline{i e^{-i \alpha} \Theta\left(i e^{-i \alpha} x, y\right)}\right\}
$$

is a projective Finsler metric with $\mathbf{K}=1$, and its projector factor is given by

$$
P(x, y)=\frac{1}{2}\left\{i e^{-i \alpha} \Theta\left(i e^{-i \alpha} x, y\right)+\overline{i e^{-i \alpha} \Theta\left(i e^{-i \alpha} x, y\right)}\right\} .
$$

Further, $F(0, y)=\cos (\alpha) \phi(y)$ and $P(0, y)=\sin (\alpha) \phi(y)$.

\section{REFERENCES}

[AIM] P. L. Antonelli, R. S. Ingarden, and M. Matsumoto, The theory of sprays and Finsler spaces with applications in physics and biology, FTPH 58, Kluwer Academic Publishers, 1993. MR 95e:53094

[Al] R. Alexander, Planes for which the lines are the shortest paths between points, Illinois J. of Math., 22(1978), 177-190. MR 82d:53042

[Alv1] J. C. Álvarez Paiva, Contact topology, taut immersions, and Hilbert's fourth problem, in "Differential and Symplectic Topology of Knots and Curves". S. Tabachnikov (Ed.). Adv. in Math. Sciences, Amer. Math. Soc., Providence, RI, 1999, 1-21. MR 2001f:53161

[Alv2] J. C. Álvarez, Symplectic geometry and Hilbert's fourth problem, preprint.

[AlFe] J. C. Álvarez Paiva and E. Fernandes, Crofton formulas in projective Finsler spaces, Electronic Research Announcements of the Amer. Math. Soc. 4(1998), 91-100. MR 99j:53097

[AlGeSm] J. C. Álvarez, I. M. Gelfand and M. Smirnov, Crofton densities, symplectic geometry and Hilbert's fourth problem, Arnold-Gelfand Mathematical Seminars, Geometry and Singularity Theory, V .I. Arnold, I. M. Gelfand, M. Smirnov, and V. S. Retakh (eds.). Birkhäuser, Boston, 1997, pp. 77-92. MR 98a:52005

[Am] R. V. Ambartzumian, A note on pseudo-metrics on the plane, Z. Wahrscheinlichkeitstheorie und verw. Gebiete, 37(1976), 145-155. MR 54:14035

[AZ] H. Akbar-Zadeh, Sur les espaces de Finsler à courbures sectionnelles constantes, Bull. Acad. Roy. Bel. Cl, Sci, 5e Série - Tome LXXXIV (1988), 281-322. MR 91f:53069

[BaRo] D. Bao and C. Robles, On Randers metrics of constant curvature, Reports on Mathematical Physics (to appear).

[BaSh] D. Bao and Z. Shen, Finsler metrics of constant curvature on the Lie group $S^{3}$, Journal of London Mathematical Society (to appear).

[BCS] D. Bao, S. S. Chern and Z. Shen, An introduction to Riemann-Finsler geometry, Springer, 2000. MR 2001g:53130

[Be1] L. Berwald, Über eine characteristic Eigenschaft der allgemeinen Räume konstanter Krümmung mit gradlinigen Extremalen, Monatsh. Math. Phys. 36(1929), 315-330.

[Be2] L. Berwald, Über die n-dimensionalen Geometrien konstanter Krümmung, in denen die Geraden die kürzesten sind, Math. Z. 30(1929), 449-469.

[Bl] W. Blaschke, Integralgeometrie 11: Zur Variationsrechnung, Abh. Math. Sem. Univ. Hamburg, 11(1936), 359-366.

[Br1] R. Bryant, Finsler structures on the 2-sphere satisfying $K=1$, Finsler Geometry, Contemporary Mathematics 196, Amer. Math. Soc., Providence, RI, 1996, 27-42. MR 97e:53128

[Br2] R. Bryant, Projectively flat Finsler 2-spheres of constant curvature, Selecta Math., New Series, 3(1997), 161-204. MR 98i:53101 
[Br3] R. Bryant, Some remarks on Finsler manifolds with constant flag curvature, Houston J. of Math. 28(2002), 221-262.

[Bu] H. Busemann, Problem IV: Desarguesian spaces, in Mathematical Developments arising from Hilbert Problems, Proc. Sympos. Pure Math. 28(1976), Amer. Math. Soc., Providence, RI, 131-141. MR 55:3940

[Fk1] P. Funk, Über Geometrien, bei denen die Geraden die Kürzesten sind, Math. Annalen 101(1929), 226-237.

[Fk2] P. Funk, Über zweidimensionale Finslersche Räume, insbesondere über solche mit geradlinigen Extremalen und positiver konstanter Krümmung, Math. Zeitschr. 40(1936), 86-93.

[Fk3] P. Funk, Eine Kennzeichnung der zweidimensionalen elliptischen Geometrie, Österreichische Akad. der Wiss. Math.-Natur., Sitzungsberichte Abteilung II 172(1963), 251269. MR 30:1460

[Ha] G. Hamel, Uber die Geometrien, in denen die Geraden die Kürtzesten sind, Math. Ann. 57(1903), 231-264.

[Hi] D. Hilbert, Mathematical Problems, Bull. Amer. Math. Soc. 37(2001), 407-436. Reprinted from Bull. Amer. Math. Soc. 8 (July 1902), 437-479.

[MaSh] M. Matsumoto and H. Shimada, The corrected fundamental theorem on the Randers spaces of constant curvature, Tensor, N.S. (to appear).

[Ok] T. Okada, On models of projectively flat Finsler spaces of constant negative curvature, Tensor, N. S. 40(1983), 117-123. MR 87c:53124

[Po] A. V. Pogorelov, Hilbert's Fourth Problem, Winston \& Wiley, New York, 1979. MR 80j:53066

[Ran] G. Randers, On an asymmetric metric in the four-space of general relativity, Phys. Rev. 59(1941), 195-199.

[Rap] A. Rapcsák, Über die bahntreuen Abbildungen metrischer Räume, Publ. Math. Debrecen, 8(1961), 285-290. MR 25:1526

[Sh1] Z. Shen, Differential Geometry of Spray and Finsler Spaces, Kluwer Academic Publishers, Dordrecht, 2001.

[Sh2] Z. Shen, Finsler metrics with $\mathbf{K}=0$ and $\boldsymbol{S}=0$, Canadian J. Math. (to appear).

[Sh3] Z. Shen, Two-dimensional Finsler metrics of constant flag curvature, Manuscripta Mathematica (to appear).

[Sh4] Z. Shen, Projectively flat Randers metrics of constant flag curvature, Math. Ann. (to appear).

[Sz] Z. I. Szabó, Hilbert's fourth problem, I, Adv. in Math. 59 (1986), 185-301. MR 88f:53113

Department of Mathematical Sciences, iUpui, 402 N. Blackford Street, IndianapoLIS, INDIANA 46202-3216

E-mail address: zshen@math.iupui.edu 